\title{
Phase imaging and the evolution of a gold-vacuum interface at atomic resolution
}

\author{
A. V. Martin, ${ }^{1}$ K. Ishizuka, ${ }^{2}$ C. Kisielowski, ${ }^{3}$ L. J. Allen ${ }^{1}$ \\ School of Physics, University of Melbourne, Victoria 3010, Australia \\ ${ }^{2}$ HREM Research Inc., Matsukazedai, Saitama 355-0055, Japan \\ ${ }^{3}$ National Center for Electron Microscopy, Ernest Orlando Lawrence Berkeley National Laboratory, Berkeley, CA 94720, USA
}

\begin{abstract}
We observe, using phase maps obtained from a focal series of images, the evolution in time of a gold-vacuum interface. What is seen is the reconfiguration and removal of whole columns of gold atoms (typically containing three to nine atoms) at the interface. These structural changes are discussed with reference to the variation in binding energy along the interface.
\end{abstract}

PACS numbers: $42.30 . \mathrm{Rx}, 61.14 .-\mathrm{x}$

\section{INTRODUCTION}

Phase retrieval based on a focal series of images has been successfully used to study structures at atomic resolution ${ }^{1-6}$ and facilitates the correction of aberrations $^{7}$. In this paper we demonstrate, by analysis of phase maps obtained from subsets of a focal series of images of a gold-vacuum interface, that significant structural changes in time can be observed. What is seen, which is quite remarkable, is the displacement of whole columns of gold atoms (typically containing three to nine atoms) at the gold-vacuum interface. The results presented here are relevant for studies of interfaces, defects and nano-particles for which significant structural change can consist of the removal, displacement or rearrangement of only a few atoms ${ }^{8-14}$.

In this investigation the iterative wave function reconstruction (IWFR) method of phase retrieval ${ }^{5}$ has been used to construct the phase maps, although we note that transport of intensity methods are also applicable ${ }^{6}$. The IWFR method starts with a guess for the phase (usually $\phi=0$ in each image plane) to provide an initial guess for the wave function in each plane in a focal series (as few as five images suffice). Each of these wave functions is propagated to a common plane (usually that which is nominally at zero defocus). At this plane an average wave function is then formed and propagated back to each image plane. The measured images in the focal series are now used to replace the intensities of the propagated wave functions. This process is iterated until some convergence criterion is satisfied. When convergence has been achieved, the method returns the averaged wave function in the common plane for the final iteration. By choosing this common plane to be the in-focus plane and by including coherent aberrations during propagation, the method returns the aberration-corrected wave function at the exit surface of the specimen. The effects of spatial and temporal incoherence can also be corrected for using envelope functions ${ }^{5}$.
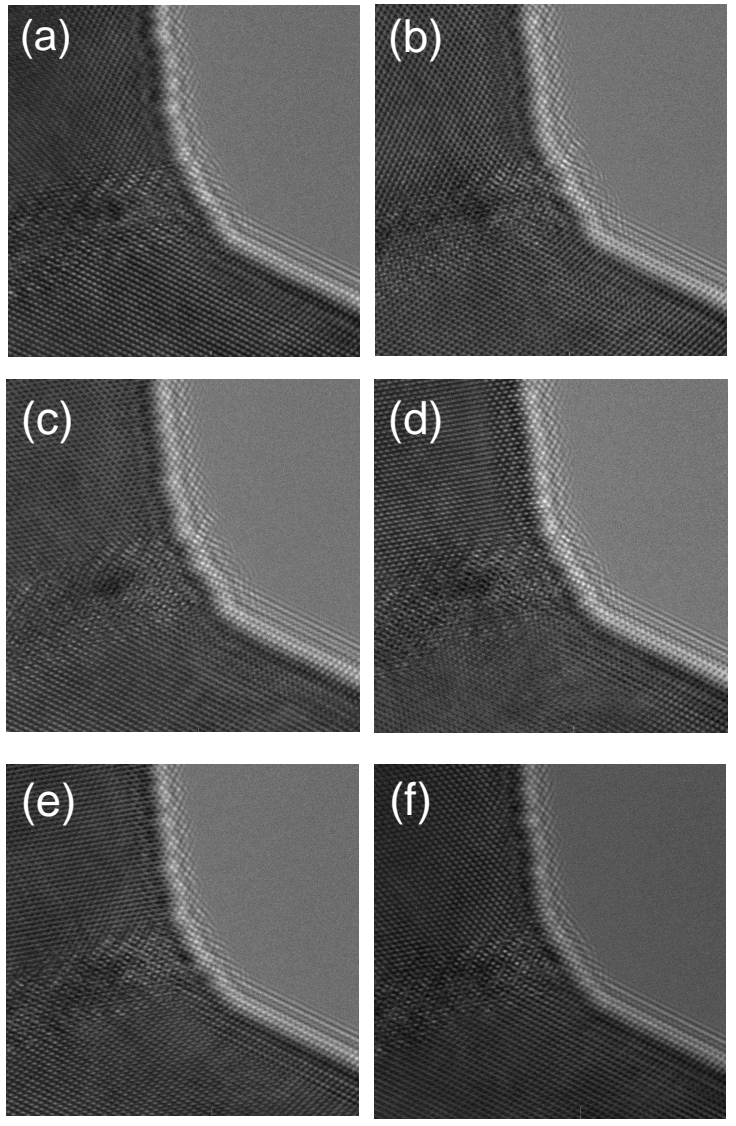

FIG. 1: A selection of images from the gold focal series at defocus values (a) $-2980 \AA$, (b) $-2884 \AA$, (c) $-2764 \AA$, (d) $-2644 \AA$, (e) $-2524 \AA$ and (f) $-2404 \AA$. The microscope parameters were: accelerating voltage $300 \mathrm{keV}$, objective aperture $1.26 \AA^{-1}, C_{\mathrm{s}}=0.6 \mathrm{~mm}$, spatial incoherence semi-angle $\beta=0.2 \mathrm{mrad}$ and temporal incoherence characterized by a defocus spread $\Delta=29 \AA$.

\section{EXPERIMENTAL DATA}

Fig. 1 shows a selection of images from a focal series of a gold-vacuum interface. The series contains twenty-five images starting with a defocus of $-2980 \AA$ (the minus sign indicates underfocus) and with a step size of $24 \AA$ 
between subsequent images. This sequence arranged according to defocus also represents the temporal order in which the images were recorded. Each image, which is $750 \times 750$ pixels, represents an area $154.1 \AA \times 154.1 \AA$ in extent. The defocus and spherical aberration $\left(C_{\mathrm{s}}=0.6\right.$ $\mathrm{mm}$ ) associated with each of these images means that structure cannot be inferred. In particular there are fringes which render the determination of the interface between the gold wafer and the interface highly ambiguous. Phase retrieval, by correcting for defocus and spherical aberration, can resolve this ambiguity, as we will now show.

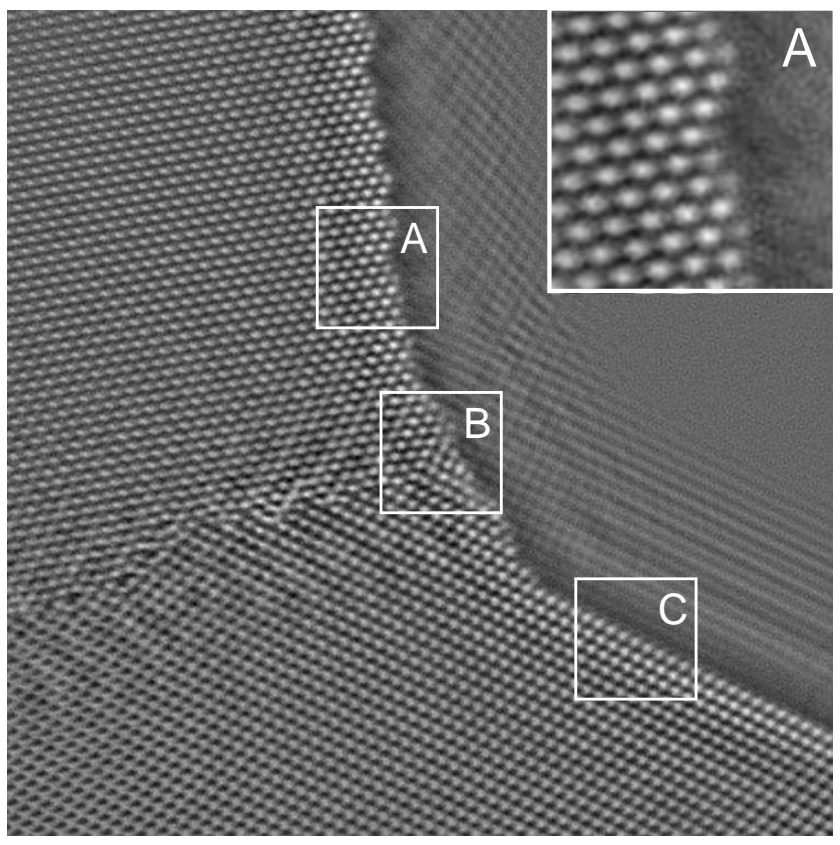

FIG. 2: Phase map of the gold-vacuum interface reconstructed from all 25 images in the focal series. Box A shows a $\langle 110\rangle$ missing row reconstruction, Box B shows a chevron defect and Box $\mathrm{C}$ shows a $\{1 \overline{1} 1\}$ straight edge. An enlarged version of Box $\mathrm{A}$ is shown in the top right hand corner.
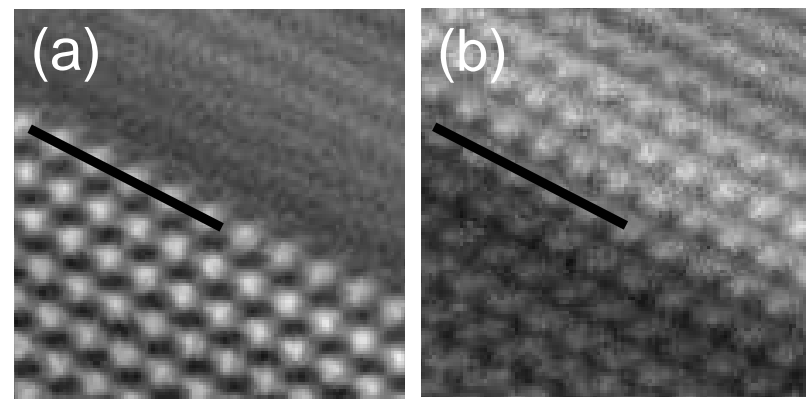

FIG. 3: Comparison of the (a) in-focus retrieved phase and (b)the first image in the focal series $(-2980 \AA)$ for Box C. The location of the interface is clearly identifiable in the retrieved phase but not in the original defocused image.

\section{PHASE RETRIEVAL RESULTS}

The result of the phase retrieval from all 25 experimental images is shown in Fig. 2. The gold specimen is seen to be composed of two crystalline regions, viewed down the $\langle 110\rangle$ axis and rotated at $90^{\circ}$ to each other. The interface between these regions forms a grain boundary. We focus on three features at the interface between the gold specimen and the vacuum. In Box A in Fig. 2 we have a "missing row" $1 \times 2$ and $1 \times 3$ reconstruction of the $\langle 110\rangle$ surface $^{15}$. In Box B we have a chevron defect ${ }^{16}$. Box $\mathrm{C}$ contains a $\{1 \overline{1} 1\}$ straight edge. This edge is particularly well defined and the gold-vacuum interface is clearly resolved at atomic resolution. Fig. 3 shows a comparison of the phase map here to the corresponding section of the original image at defocus $-2980 \AA$, where the edge cannot be correctly located.

However, as can be seen in the enlarged version of Box $\mathrm{A}$ in Fig. 2, the edge is not as clearly determined in this region. There is a suggestion of several fainter columns on the edge. Closer examination of the chevron defect in Box B reveals similar indistinctness. We will now demonstrate that this is due to structural changes at the edge which occur on a time scale shorter than the time taken to acquire the focal series. To that end we perform phase reconstruction on subsets of the focal series. With twenty-five defocused images it is possible to take twentyone retrievals using five consecutive images in the series. Hence, the first retrieval uses images 1-5, the second uses images 2-6 and so on.

Fig. 4 compares retrieved phases for the three regions of interest shown in Fig. 2. Fig. 4 (a) and (b) show, in the regions highlighted, an instance of an atomic column appearing and two other columns disappearing along the missing row reconstruction. The structure of this region of the interface is not stable in the presence of the beam and atomic columns can be removed or relocated along the interface. At the chevron defect, Fig. 4 (c)-(f), we can see several atomic columns disappearing steadily during the acquisition of the focal series, indicating that this defect is even less stable. Fig. $4(\mathrm{~g})$ and (h) show two phases for the $\{1 \overline{1} 1\}$ straight edge where no structural change is observed.

\section{DISCUSSION}

The results presented in the Sec. III suggest that there is a significantly greater probability of removing an atom from the missing row reconstruction or the chevron defect than from the $\{1 \overline{1} 1\}$ straight edge. This is consistent with atoms along the $\{1 \overline{1} 1\}$ straight edge being more tightly bound than those in the missing row reconstruction or along the chevron defect. How great is this difference in binding energies? The binding of face-centered cubic metals is mediated by localized d-shell electrons and the repulsion of nearest neighbor ions, as modeled by a tight-binding second-moment approximation ${ }^{17,18}$. In 

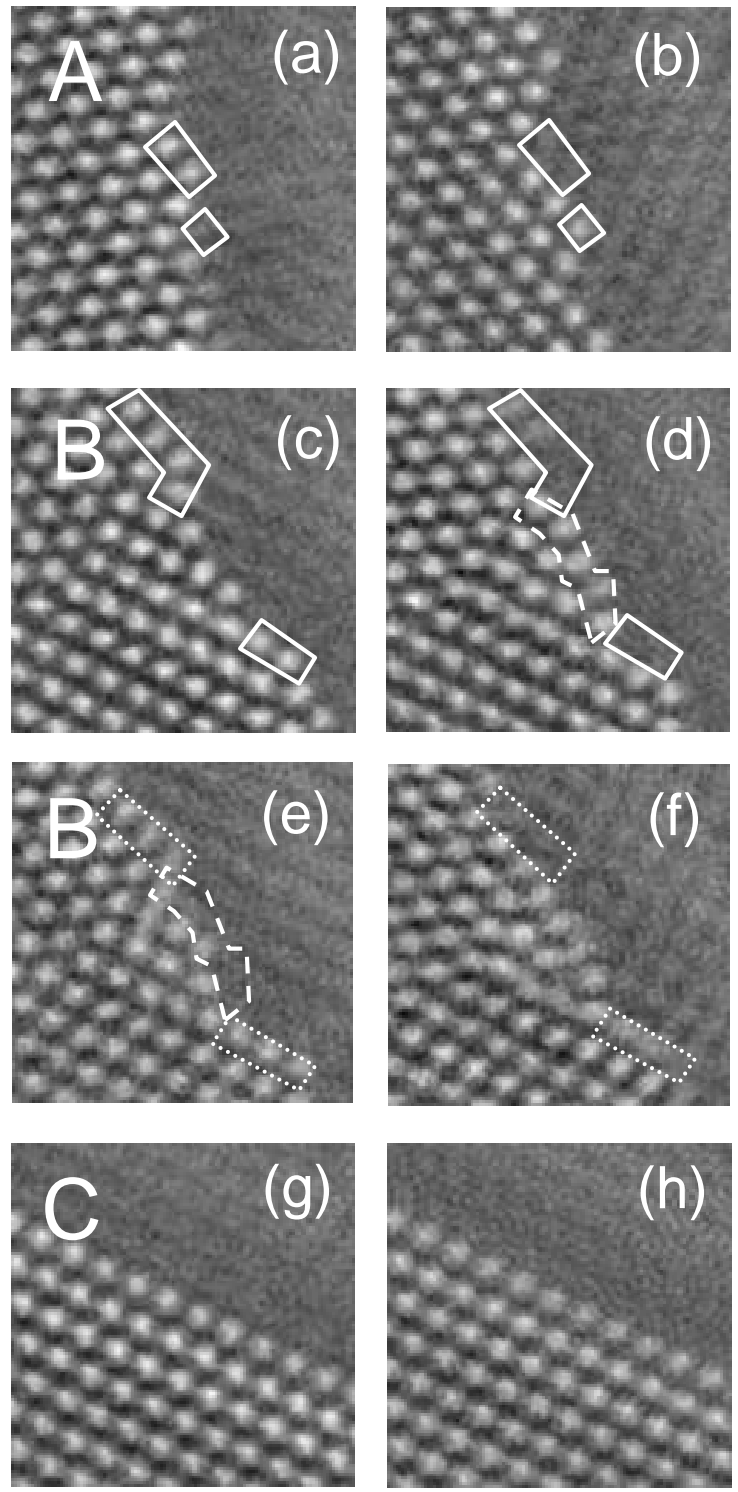

FIG. 4: (a) The phase retrieved from focal series images 610 , while (b) was retrieved from images 12-16 - they show the disappearance and appearance of atomic columns at the missing row reconstruction (Box A). (c) The phase retrieved from images 2-6, (d) from images 10-14, (e) from images 16-20, and (f) from images 21-25 - they show the repeated removal of atomic columns from the chevron defect (Box B). (g) The phase retrieved from images 1-5 and $(\mathrm{h})$ from images 15-20 they show the stability of the $\{1 \overline{1} 1\}$ straight edge (Box C). A common highlighted region in two phase maps indicates the location of a structural change.

particular, this has been used to study the properties of the chevron defect ${ }^{16}$. Using parameters fitted to the bulk properties of gold ${ }^{18}$ the binding energies of atoms at different parts of our interface were estimated. Along the $\{1 \overline{1} 1\}$ straight edge (Box C), an atom in the middle of a column has nine nearest neighbors and we estimate its binding energy to be $3.6 \mathrm{eV}$. Hence atoms along this interface are bound almost as strongly as those in the bulk (where the binding energy is $3.8 \mathrm{eV}^{19}$ ). An atom bound along the missing row reconstruction has at most five nearest neighbors, and the estimate of the binding energy is $3.0 \mathrm{eV}$. The binding energies of atoms in the chevron defect are harder to estimate as the number of nearest neighbors and the inter-atomic distances vary from atom to atom. We would expect them to be bound with similar or less strength than the atoms on the missing row reconstruction ${ }^{16}$.

The probability of ejecting an atom from the surface can be determined from the cross section of a relativistic electron scattered from a nucleus via the Coulomb interaction. This cross section, which includes electron spin, has been well studied ${ }^{20,21}$ and is the basis of sputtering models of sample thinning ${ }^{22}$. To determine the probability of ejecting an atom, this cross section is integrated over the range of angles for which the energy transferred to the atom is greater than the binding energy. The direction of the momentum transfer has not been restricted which is a reasonable assumption for atoms on the interface of a thin specimen ${ }^{22}$. The energy transferred to the atom by an electron scattered through angle $\theta$ is given by

$$
E=E_{\max } \sin ^{2}(\theta / 2)
$$

where $E_{\max }$ is the maximum possible energy transfer to be determined from relativistic kinematics ${ }^{22}$. For 300 $\mathrm{keV}$ incident electrons the maximum energy that can be transferred to the atom is $4.3 \mathrm{eV}$. This is sufficient to eject an atom from any location along the interface, as well as atoms from the entrance and exit surfaces. The total cross section for electrons that have ejected an atom was calculated approximately ${ }^{21}$ for the binding energies of the straight and missing row surfaces. They were then combined with the area of the detector and the total number of electrons incident during the recording of one image to determine the probability of losing an atom when one image is taken. The results are shown in Table I.

TABLE I: Binding energies and cross sections for the removal of an atom from two different edges. The rightmost column shows the total probability for removing an atom during one image exposure for our focal series.

\begin{tabular}{cccc}
\hline \hline location & $E_{b}(\mathrm{eV})$ & $\sigma\left(\AA^{2}\right)$ & probability \\
\hline$\{1 \overline{1} 1\}$ straight edge & 3.6 & $1.4 \times 10^{-6}$ & 0.1 \\
missing row & 3.0 & $4.6 \times 10^{-6}$ & 0.3 \\
\hline \hline
\end{tabular}

The differences in binding energy lead to a significant difference in the probability of losing an atom during the recording of an image. There is about a 30\% chance of losing the most weakly bound atom from the the missing row interface. Since we estimate that columns can contain as few as three atoms, this probability is consistent with our observation of column removal and relocation along this edge. By comparison, the atoms along the $\{1 \overline{1} 1\}$ straight edge have only a $10 \%$ chance of being 
ejected during the exposure of one image. This suggests that some atoms are also being ejected from this interface, even though no structural change is observed in the retrieved phases. Since we estimate that there can be up to nine atoms in a column, the removal of such a column during one image exposure is unlikely. Furthermore, should multiple atoms be removed from a column at the $\{1 \overline{1} 1\}$ straight edge, the edge may relax to produce the same observed structure before and after. It should be noted that our estimates only include the electrons incident on the sample during the recording of an image. But the specimen remains in the beam when the image is read out and the microscope adjusted to a new defocus. Hence, there is further opportunity for structural change between images, which would contribute to our observation of the removal of several atoms in an atomic column.

In this paper we have discussed the removal of whole columns of atoms at the gold-vacuum interface. In principle more refined measurements and analysis than that presented here could definitively identify the partial removal of a column of atoms.

\section{CONCLUSION}

When considering the possibility of the sample changing structurally we must identify those features, like defects and interfaces, where lower binding energy leads to an increased susceptibility to the incident beam. For phase imaging, a significant susceptibility should be gauged by whether the removal of single atoms would influence the interpretation of the retrieved phase.

The probability of structural changes occurring is dependent on the total incident flux, and hence on the recording and read-out time of one image, in our experiment of the order of a second. However, faster cameras with recording and read-out times of the order 0.01 seconds, envisaged in the next generation of leading-edge microscopes, may allow these sensitive structures to be studied unchanged by the incident beam.

It has been shown that when structural change occurs during a focal series of measurements, phase retrieval returns an averaged phase. This may complicate the interpretation of nano-sized structures like defects and interfaces, whose structure is not necessarily stable in the presence of the incident beam. However, the temporal evolution of such structures can be observed by phase retrieval from subsets of the focal series, which will be an important tool for the study of phase objects in time.

\section{Acknowledgments}

A. V. Martin and L. J. Allen acknowledge helpful discussions with S. D. Findlay. L. J. Allen acknowledges support by the Australian Research Council. The authors acknowledge support of the National Center for Electron Microscopy, Lawrence Berkeley Lab, which is supported by the U.S. Department of Energy under Contract \# DE-AC02-05CH11231.
1 W. Coene, G. Janssen, M. O. de Beeck, and D. Van Dyck, Phys. Rev. Lett. 69, 3743 (1992).

${ }^{2}$ C. L. Jia and A. Thust, Phys. Rev. Lett. 82, 5052 (1999).

${ }^{3}$ C. Kisielowski, C. J. D. Hetherington, Y. C. Wang, R. Kilaas, M. A. O'Keefe, and A. Thust, Ultramicroscopy 89, $243(2001)$

${ }^{4}$ M. A. O'Keefe, C. J. D. Hetherington, Y. C. Wang, E. C. Nelson, J. H. Turner, C. Kisielowski, J.-O. Malm, R. Mueller, J. Ringnalda, M. Pan, et al., Ultramicroscopy 89, 215 (2001).

${ }^{5}$ L. J. Allen, W. McBride, N. L. O'Leary, and M. P. Oxley, Ultramicroscopy 100, 91 (2004).

${ }^{6}$ K. Ishizuka and B. Allman, J. Electron Microsc. 53, 191 (2005).

7 L. J. Allen, M. P. Oxley, and D. Paganin, Phys. Rev. Lett. 87, $123902(2001)$

8 L. D. Marks and D. J. Smith, Nature 303, 316 (1983).

${ }^{9}$ S. Iijima and T. Ichihashi, Jpn. J. Appl. Phys. 24, L125 (1985).

10 J.-O. Bovin, R. Wallenberg, and D. J. Smith, Nature 317, 47 (1985).

11 D. J. Smith, A. K. Petford-Long, L. R. Wallenberg, and J.-O. Bovin, Science 233, 872 (1986).
12 D. A. Muller and M. J. Mills, Mat. Sci. Eng. A260, 12 (1999).

13 C. Kisielowski, E. Principe, B. Freitag, and D. Hubert, Physica B 308-310, 1090 (2001).

14 J.-G. Lee, J. Lee, T. Tanaka, and H. Mori, Phys. Rev. Lett. 96, 075504 (2006)

15 K.-M. Ho and K. P. Bohmen, Phys. Rev. Lett. 59, 1833 (1987).

16 T. Radetic, F. Lancon, and U. Dahmen, Phys. Rev. Lett. 89, 085502 (2002).

17 V. Rosato, M. Guillope, and B. Legrand, Philos. Mag. A 59, 321 (1989).

18 T. Deutsch, P. Bayle, F. Lancon, and J. Thibault, J. Phys. Condens. Matter 7, 6407 (1995).

19 C. Kittel, Introduction to Solid State Physics (John Wiley, New Jersey, 2005), eighth ed.

20 N. F. Mott, Proc. R. Soc. London A 78, 425 (1929).

${ }^{21}$ W. McKinnley and H. Feshbach, Phys. Rev. 74, 1759 (1948).

22 R. F. Egerton, F. Wang, and P. A. Crozier, Microsc. Microanal. 12, 1 (2006). 